\title{
Stochastic classical field model for polariton condensates
}

\author{
Michiel Wouters and Vincenzo Savona \\ Insitute of Theoretical Physics, Ecole Polytechnique Fédérale de Lausanne (EPFL), CH-1015 Lausanne, Switzerland \\ (Received 27 November 2008; revised manuscript received 12 February 2009; published 6 April 2009)
}

\begin{abstract}
We use the truncated Wigner approximation to derive stochastic classical field equations for the description of polariton condensates. Our equations are shown to reduce to the Boltzmann equation in the limit of low polariton density. Monte Carlo simulations are performed to analyze the momentum distribution and the firstand second-order coherences when the particle density is varied across the condensation threshold.
\end{abstract}

DOI: 10.1103/PhysRevB.79.165302

PACS number(s): 71.36.+c, 05.70.Ln, 11.30.Qc

\section{INTRODUCTION}

Condensates of microcavity polaritons ${ }^{1}$ are a solid-state realization of the two-dimensional Bose gas. Their successful creation relies on the peculiar nature of the microcavity polariton quasiparticle that combines a very light effective mass (high quantum degeneracy temperature) with interparticle interactions that provide efficient relaxation. The formation of spontaneous coherence in these systems is now routinely achieved in several laboratories. ${ }^{2-5}$

One crucial difference between polariton condensates and other realizations of the two-dimensional Bose gas such as liquid ${ }^{4} \mathrm{He}$ films ${ }^{6}$ and tightly confined ultracold atomic gases ${ }^{7}$ comes from the finite lifetime of the microcavity polaritons of the order of a few ps. In order to compensate for the polariton losses, new particles can be continuously injected into the microcavity. The resulting steady state is not a thermal equilibrium one; still it shows features expected for an equilibrium BEC. For example, the tail of the momentum distribution can in many cases be fitted by an exponential Maxwell-Boltzmann decay. The lack of full thermalization is already clear from the fact that the extracted temperature is in general not equal to the temperature of the reservoir constituted by the semiconductor lattice. ${ }^{2-4}$

Effects that have no counterpart at equilibrium have been observed in polariton condensates. For example, the condensate state can depend dramatically on the size of the excitation spot: ${ }^{8,9}$ in the case of a large pump spot the usual condensation around zero momentum is observed, instead for a small excitation spot the condensation occurs on a ring in momentum space. This difference has been explained within a mean-field theory based on the Gross-Pitaevskii equation, including driving and dissipation..$^{10}$ More recently, another remarkable phenomenon related to the flow in a continuously pumped polariton condensate was observed experimentally: ${ }^{11}$ vortices are spontaneously created in polariton condensates without setting the system into rotation. A theoretical interpretation of this effect was given in the framework of the generalized Gross-Pitaevskii equation. In a significant fraction of random landscape realizations the polariton condensate contains a vortex. A related prediction was made by Keeling and Berloff: ${ }^{12}$ they found that a rotating vortex lattice can be spontaneously generated in a large regular trap.

The above-mentioned phenomena can be understood within a mean-field theory, i.e., a theory where the quantum polariton field is replaced by a classical field. In this approximation, all information on the fluctuations is however lost. Since we deal with a two-dimensional system, the physics of fluctuations in polariton condensates is in analogy with equilibrium systems expected to be very rich $^{7,13,14}$ and the question arises, for example, to what extent the physics related to the Berezinskii-Kosterlitz-Thouless survives the driving and dissipation of polariton condensates.

Experimentally, the fluctuations of the polariton condensates have been investigated under the form of the first- and second-order coherence functions. In the first-order equal time spatial correlation function, long-range correlations were observed above the stimulation threshold for condensation. ${ }^{3,15}$ Other correlation functions include the temporal first- ${ }^{5}$ and second-order coherences. ${ }^{5,16,17}$

The semiclassical Boltzmann equation ${ }^{18-20}$ provides a theoretical description of the first-order spatial coherence, which is the Fourier transform of the momentum distribution. Including the details of the relaxation mechanisms, this formalism is expected to give a reliable estimate for the required polariton density to achieve condensation. A Boltzmann description that takes the polarization degree of freedom into account can be found in Ref. 21. Above the condensation threshold, the random phase approximation contained in the Boltzmann equation breaks down and more sophisticated techniques should be used. Schemes that have been implemented in the literature involve the separation of the condensate mode from the excited states ${ }^{22}$ and a generalization of the Boltzmann equation that includes the coherences within a Bogoliubov approximation. ${ }^{23}$

One of the remarkable consequences of the nonequilibrium nature of the polariton condensates is that the collective excitation spectrum is changed at low wave vectors: a diffusive instead of soundlike character is found for the lowenergy phase modes. This dispersion of elementary excitations was found in a theoretical description based on a Keldysh Green's-function technique worked out by Littlewood et al. ${ }^{24}$ The same spectrum is straightforwardly recovered by linearizing the generalized Gross-Pitaevskii equation around a steady state, ${ }^{25}$ a calculation that is easily extended to spatially nonuniform situations.

It is well known from quantum optics and the theory of weakly interacting Bose gases that fluctuations can be included by introducing a stochastic element in the GrossPitaevskii equation. ${ }^{26-31}$ For polariton systems in the parametric oscillation regime, such a method was used in Ref. 32. One of the great advantages of these so called classical 


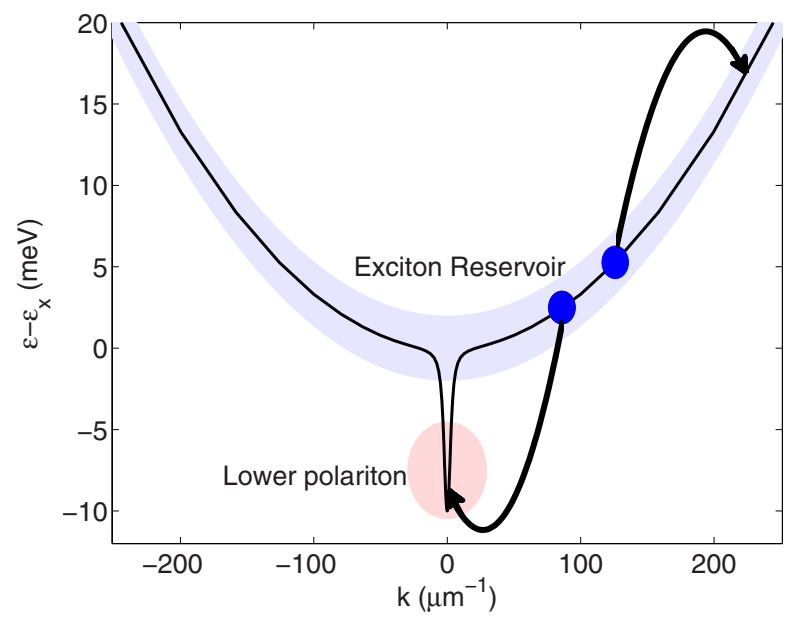

FIG. 1. (Color online) Sketch of the separation of phase space in two regions: the lower polariton and the exciton reservoir. Scattering from the exciton reservoir into the lower polariton region replenishes the lower polaritons.

field methods is that the nonuniformity of a system does not introduce any appreciable extra cost in their numerical implementation. A second advantage is that practical numerical calculations do not require a perturbative expansion around a condensed state and can even be applied to study physics related to the condensation phase transition. ${ }^{33,34} \mathrm{Fi}-$ nally, these methods can describe the evolution of the system in real time so that information on both the steady state and transients can be obtained. The latter can be of particular use to model experiments that are performed under pulsed excitation. $^{2}$

Due to the approximations involved in the classical field methods, they cannot describe the particles up to arbitrary large momenta, where quantum effects (most notably spontaneous scattering) are dominant. ${ }^{26}$ In this respect, polariton condensates are very well suited for a classical field description because, as illustrated in Fig. 1, the phase space can be naturally divided into two parts: (i) a low-energy polaritonic region with a small effective mass of about $10^{-4}$ times the bare electron mass that allows to reach quantum degeneracy at densities of $10^{8} \mathrm{~cm}^{-2}$ at a temperature of $10 \mathrm{~K}, 8$ orders of magnitude larger than the degeneracy temperature of ultracold atoms at comparable densities, and (ii) a high-energy excitonic "reservoir" region with a high effective mass that under typical experimental densities behaves as an incoherent classical gas. The role of the two subsystems is very different: the polaritonic field is the quantity of experimental interest because it is easily accessible in photoluminescence experiments and can be driven into the quantum degenerate regime. The role of the reservoir is to replenish the polariton region through relaxation.

We will present in this paper a set of classical field equations for the (Wigner distribution function of the) polariton dynamics coupled to the exciton reservoir and apply them to calculate the equal time first- and second-order coherence functions across the condensation threshold. For simplicity, we have not included the polarization degree of freedom. At thermal equilibrium, the magnitude of fluctuations can be parametrized by a single quantity, the temperature. For a weakly interacting system, this temperature can be extracted by fitting the tail of the momentum distribution with a Maxwellian curve. Experiments have shown that the MaxwellBoltzmann distribution is also recovered for the tail of the out of equilibrium polariton distribution ${ }^{2-4}$ and has even been experimentally observed in the case of weak coupling lasing. ${ }^{35}$ We will show that out of equilibrium, the universal characterization of fluctuations by a temperature parameter breaks down. We will point out several crucial aspects of the condensate-reservoir interactions that affect the correlation functions without changing the tail of the momentum distribution.

We start by presenting the model Hamiltonian for the nonresonantly excited polariton system in Sec. II. It is shown in Sec. III how a master equation for the lower polariton (LP) field can be derived and how to solve it within the truncated Wigner approximation in Sec. IV. The reservoir dynamics is discussed in Sec. V. In Sec. VI, we discuss the relation between our model and the Boltzmann equation. Numerical Monte Carlo results are presented in Sec. VII. Conclusions are drawn in Sec. VIII.

\section{HAMILTONIAN}

In order to treat the two regions of polaritonic phase space with a very different character, we replace the original Hamiltonian by a Hamiltonian for polaritons and excitons that are annihilated by the operators $\psi(\mathbf{x})$ and $\phi(\mathbf{x})$, respectively. In terms of these annihilation operators, our model Hamiltonian reads as

$$
H=\int d \mathbf{x}\left[H_{\mathrm{LP}}(\mathbf{x})+H_{R}(\mathbf{x})+H_{R, \mathrm{LP}}(\mathbf{x})\right] .
$$

The lower polariton Hamiltonian density is the usual

$$
H_{\mathrm{LP}}(\mathbf{x})=\psi^{\dagger}(\mathbf{x}) \epsilon_{\mathrm{LP}}(-i \nabla) \psi(\mathbf{x})+\frac{g}{2} \psi^{\dagger}(\mathbf{x}) \psi^{\dagger}(\mathbf{x}) \psi(\mathbf{x}) \psi(\mathbf{x}),
$$

where $g$ quantifies the strength of the polariton-polariton interactions, that is well approximated by a zero-range potential. The exciton reservoir Hamiltonian is given by

$$
H_{R}(\mathbf{x})=\phi^{\dagger}(\mathbf{x}) \epsilon_{X}(-i \nabla) \phi(\mathbf{x})+\frac{g}{2} \phi^{\dagger}(\mathbf{x}) \phi^{\dagger}(\mathbf{x}) \phi(\mathbf{x}) \phi(\mathbf{x}) .
$$

Both the lower polariton and exciton dispersions are approximated by parabolic functions close to their minimum $\epsilon_{\mathrm{LP}, X}(k)=\epsilon_{\mathrm{LP}, X}+k^{2} / 2 m_{\mathrm{LP}, X}$. In the polariton/exciton basis, the exciton-exciton Coulomb scattering gives rise to various coupling terms. The relevant ones are

$$
H_{R, \mathrm{LP}}(\mathbf{x})=H_{R, \mathrm{LP}}^{\text {loss }}(\mathbf{x})+H_{R, \mathrm{LP}}^{\mathrm{gain}}(\mathbf{x})+H_{R, \mathrm{LP}}^{\mathrm{mf}}(\mathbf{x}) .
$$

Lower polaritons are created by the term

$$
H_{R, \mathrm{LP}}^{\text {gain }}(\mathbf{x})=g \phi^{\dagger}(\mathbf{x}) \phi(\mathbf{x}) \phi(\mathbf{x}) \psi^{\dagger}(\mathbf{x}),
$$

whereas they are destroyed by 


$$
H_{R, \mathrm{LP}}^{\text {loss }}(\mathbf{x})=g \phi^{\dagger}(\mathbf{x}) \phi^{\dagger}(\mathbf{x}) \phi(\mathbf{x}) \psi(\mathbf{x}) .
$$

Mean-field shifts of the lower polaritons due to the excitons in the reservoir and vice versa are described by the Hamiltonian

$$
H_{R, \mathrm{LP}}^{\mathrm{mf}}(\mathbf{x})=g \phi^{\dagger}(\mathbf{x}) \phi(\mathbf{x}) \psi^{\dagger}(\mathbf{x}) \psi(\mathbf{x}) .
$$

Note that we have in fact extended phase space by introducing two particles: the excitonic phase space is extended down to $k=0$ and the polaritonic phase space to arbitrarily large momenta. Both extensions however add a very few states in the physically relevant regions.

\section{MASTER EQUATION}

In order to take advantage of the incoherent nature of the excitons in the reservoir, we will trace them out from the dynamics and obtain a quantum equation for the LP field alone. The LP field dynamics can be studied through the Liouville equation for the density matrix

$$
\dot{\rho}=-i[H, \rho] \text {. }
$$

Going through the usual steps in the derivation of the master equation in quantum optics, the master equation for the full density matrix reads in the second Born approximation in $H_{R, \mathrm{LP}}$

$$
\begin{aligned}
\rho(t)= & \rho\left(t_{0}\right)-i \int_{t_{0}}^{t} d t^{\prime}\left[H_{R, \mathrm{LP}}\left(t^{\prime}\right), \rho_{\mathrm{LP}}\left(t^{\prime}\right)\right] \\
& -\int_{t_{0}}^{t} d t^{\prime} \int_{t_{0}}^{t^{\prime}} d t^{\prime \prime}\left[H_{R, \mathrm{LP}}\left(t^{\prime}\right),\left[H_{R, \mathrm{LP}}\left(t^{\prime \prime}\right), \rho\left(t^{\prime \prime}\right)\right]\right],
\end{aligned}
$$

where $H_{R, \mathrm{LP}}(t)$ is in the interaction picture with respect to the Hamiltonian $H_{0}=H_{\mathrm{LP}}+H_{R}$.

Taking the trace of this equation over the reservoir degrees of freedom gives the desired master equation for the reduced density matrix $\rho_{\mathrm{LP}}$ of the lower polariton subsystem. The second term on the right-hand side vanishes when the trace over the reservoir is taken, so we only have to analyze the third one. It consists of terms such as

$$
\operatorname{Tr}_{R}\left\{H_{R, \mathrm{LP}}^{\mathrm{gain} / \text { loss }} H_{R, \mathrm{LP}}^{\text {gain } / \text { loss }} \rho\right\}
$$

where

$$
\operatorname{Tr}_{R}\left\{H_{R, \mathrm{LP}}^{\text {gain }} H_{R, \mathrm{LP}}^{\text {gain }} \rho_{\mathrm{LP}}\right\}=\operatorname{Tr}_{R}\left\{H_{R, \mathrm{LP}}^{\text {loss }} H_{R, \mathrm{LP}}^{\text {loss }} \rho_{\mathrm{LP}}\right\}=0 .
$$

A nonzero term is, e.g., given by

$$
R_{1}=\int d t^{\prime} d t^{\prime \prime} \operatorname{Tr}_{R}\left\{H_{R, \mathrm{LP}}^{\mathrm{gain}}\left(t^{\prime}\right) H_{R, \mathrm{LP}}^{\mathrm{loss}}\left(t^{\prime \prime}\right) \rho\left(t^{\prime \prime}\right)\right\}
$$

In order to work out the trace over the reservoir, we introduce relative and center of mass coordinates

$$
\mathbf{X}=\frac{\mathbf{x}^{\prime}+\mathbf{x}^{\prime \prime}}{2}, \quad \mathbf{x}=\mathbf{x}^{\prime}-\mathbf{x}^{\prime \prime}
$$

$$
T=\frac{t^{\prime}+t^{\prime \prime}}{2}, \quad t=t^{\prime}-t^{\prime \prime}
$$

As in Ref. 30, we define the Wigner transform of the reservoir propagator as

$$
\begin{aligned}
F^{W}(\mathbf{X}, \mathbf{k}, T, \omega)= & \int d t d \mathbf{x} e^{i \omega t} e^{-i \mathbf{k} \mathbf{x}} \operatorname{Tr}_{R}\left\{\phi^{\dagger}(\mathbf{X}+\mathbf{x} / 2, T\right. \\
& +t / 2) \phi(\mathbf{X}-\mathbf{x} / 2, T-t / 2) \rho\} .
\end{aligned}
$$

With the inverse transformation, we obtain for $R_{1}$ defined in Eq. (12)

$$
\begin{aligned}
R_{1}= & \frac{1}{\Omega^{3}} \sum_{\mathbf{k}_{1,2,3}} \int d \mathbf{X} d \mathbf{x} e^{i(\Delta \mathbf{k} \cdot \mathbf{x}-\Delta \epsilon t)} \Pi_{f}\left(\mathbf{X}, \mathbf{k}_{1,2,3}\right) \psi^{\dagger}(\mathbf{X}+\mathbf{x} / 2, T \\
& +t / 2) \psi(\mathbf{X}-\mathbf{x} / 2, T-t / 2) \rho(T-t / 2),
\end{aligned}
$$

where $\Omega$ is the area of our system, $\Delta \mathbf{k}=\mathbf{k}_{2}+\mathbf{k}_{3}-\mathbf{k}_{1}$, $\Delta \epsilon=\epsilon_{X}\left(\mathbf{k}_{2}\right)+\epsilon_{X}\left(\mathbf{k}_{3}\right)-\epsilon_{X}\left(\mathbf{k}_{1}\right)$, and $\Pi_{f}\left(\mathbf{X}, \mathbf{k}_{1,2,3}\right)$ is a typical Boltzmann collision rate (density in phase space),

$$
\Pi_{f}\left(\mathbf{X}, \mathbf{k}_{1,2,3}\right)=f\left(\mathbf{X}, \mathbf{k}_{1}, T\right)\left[f\left(\mathbf{X}, \mathbf{k}_{2}, T\right)+1\right]\left[f\left(\mathbf{X}, \mathbf{k}_{3}, T\right)+1\right] .
$$

We have used the quasiparticle approximation ${ }^{36}$

$$
F^{W}(\mathbf{X}, \mathbf{k}, T, \omega)=(2 \pi i) \delta\left[\omega-\epsilon_{X}(\mathbf{k})\right] f(\mathbf{X}, \mathbf{k}, T) .
$$

The time evolution of the LP field operators is approximately given by

$$
\psi^{\dagger}(\mathbf{X}+\mathbf{x} / 2, T+t / 2) \simeq \frac{1}{\Omega} \sum_{\mathbf{Q}} e^{i \mathbf{Q}(\mathbf{X}+\mathbf{x} / 2)} e^{i \epsilon_{\mathrm{LP}}(\mathbf{Q}) t} \psi^{\dagger}(\mathbf{Q}, T-t / 2),
$$

where the interaction shift in the frequency of $\psi_{\mathrm{Q}}$ was neglected. The exponential $e^{i \mathbf{Q x}}$ can be combined with the exponential in Eq. (16). Because the typical reservoir momentum is much larger than the typical lower polariton momentum (see Fig. 1), this factor is negligible. For the same reason, also the $\mathbf{x}$ in the second field operator in Eq. (16) can be neglected. If we then also assume that the density matrix is slowly varying on the microscopic time scale $t$, the integral over the relative time imposes energy conservation for the scattering process. We can then finally rewrite Eq. (12) as

$$
\begin{aligned}
R_{1}\left[\epsilon_{\mathrm{LP}}(\mathbf{q})\right]= & \pi g^{2} T \int d \mathbf{X} \frac{1}{\Omega^{3}} \sum_{\mathbf{k}_{1}, \mathbf{k}_{2}, \mathbf{k}_{3}, \mathbf{Q}} \delta_{\Delta \mathbf{k}} \delta_{\Delta \epsilon+\epsilon_{\mathrm{LP}}(\mathbf{q})} \Pi_{f}\left(\mathbf{X}, \mathbf{k}_{1,2,3}\right) \\
& \times \psi_{\mathbf{Q}}^{\dagger}(T) \psi_{\mathbf{X}}(T) \rho(T) .
\end{aligned}
$$

The main simplifying assumption of the model consists now of assuming that expression (20) is a function of the total reservoir density $n_{R}$ and the energy $\epsilon_{\mathrm{LP}}(\mathbf{q})$ only. This comes down to the assumption of a steady-state distribution of the reservoir excitons among the different $\mathbf{k}$ states.

Working out the trace over the reservoir in Eq. (9) yields gain and loss terms for the lower polariton field from the collisions involving reservoir excitons. Collecting all terms, we obtain 


$$
\frac{d}{d t} \rho_{\mathrm{LP}}(t)=-i\left[H_{\mathrm{LP}}, \rho_{\mathrm{LP}}\right]+K_{\mathrm{in}}\left(\rho_{\mathrm{LP}}\right)+K_{\mathrm{out}}\left(\rho_{\mathrm{LP}}\right),
$$

where the density matrix evolves under the inscattering as

$$
\begin{aligned}
K_{\mathrm{in}}(\rho)= & \frac{1}{2} \sum_{\mathbf{q}} \int d \mathbf{x} R_{\mathrm{in}}\left[n_{R}, \epsilon_{\mathrm{LP}}(\mathbf{q})\right] \\
& \times\left[e^{i \mathbf{q} \mathbf{x}} \psi^{\dagger}(\mathbf{x}) \rho \psi(\mathbf{q})-e^{i \mathbf{q} \mathbf{x}} \psi(\mathbf{q}) \psi^{\dagger}(\mathbf{x}) \rho+\text { H.c. }\right],
\end{aligned}
$$

and under outscattering as

$$
\begin{aligned}
K_{\text {out }}(\rho)= & \frac{1}{2} \sum_{\mathbf{q}} \int d \mathbf{x} R_{\text {out }}\left[n_{R}, \epsilon_{\mathrm{LP}}(\mathbf{q})\right]\left[e^{i \mathbf{q x}} \psi(\mathbf{q}) \rho \psi^{\dagger}(\mathbf{x})\right. \\
& \left.-e^{i \mathbf{q x}} \psi^{\dagger}(\mathbf{x}) \psi(\mathbf{q}) \rho+\text { H.c. }\right] .
\end{aligned}
$$

The rates $R_{\text {in/out }}$ are given by the usual semiclassical Boltzmann rates. Neglecting stimulated processes in the reservoir, $R_{\text {in }}$ and $R_{\text {out }}$ depend on the reservoir density, respectively, as $n_{R}^{2}$ and $n_{R}$. We therefore write

$$
\begin{aligned}
R_{\text {in }}\left[n_{R}, \epsilon_{\mathrm{LP}}(\mathbf{q})\right] & =n_{R}^{2} R_{\text {in }}\left[\epsilon_{\mathrm{LP}}(\mathbf{q})\right], \\
R_{\text {out }}\left[n_{R}, \epsilon_{\mathrm{LP}}(\mathbf{q})\right] & =n_{R} R_{\text {out }}\left[\epsilon_{\mathrm{LP}}(\mathbf{q})\right] .
\end{aligned}
$$

Actually, another loss mechanism for the lower polariton field is present: leakage of the photon out of the imperfect microcavity mirrors that gives a finite linewidth $\gamma$ to the lower polariton. This loss mechanism has a negligible energy and momentum dependence and can be added to the model by simply adding the constant term $\gamma$ to $R_{\text {out }}$.

\section{WIGNER}

An exact solution of the master equation (21) is not possible, but numerical progress can be made by the use of quasiprobability distributions from quantum optics. In the presence of dissipation, the Wigner distribution function is believed to give robust results (see Ref. 37, pp. 115 and 124). This method has been applied to study Bose-Einstein Condensation (BEC) aspects of parametrically generated signal polariton in microcavities in Ref. 32.

The Wigner distribution function is a quasiprobability distribution defined on the space spanned by the complex valued functions $\psi(\mathbf{x})$. In order to avoid ambiguity, we will use below an explicit "hat" notation for the quantum field operator $\hat{\psi}(\mathbf{x})$.

In terms of the density matrix, the Wigner distribution function is defined as

$$
\begin{aligned}
P_{W}[\psi(\mathbf{x})]= & \frac{1}{\pi^{2}} \int d^{2} \lambda(\mathbf{x}) \exp \left[\psi(\mathbf{x}) \lambda(\mathbf{x})^{*}-\psi(\mathbf{x})^{*} \lambda(\mathbf{x})\right] \\
& \times \frac{1}{\pi} \int d^{2} \alpha(\mathbf{x})\langle\alpha(\mathbf{x})| \rho \\
& \times \exp \left[\lambda(\mathbf{x}) \hat{\psi}^{\dagger}(\mathbf{x})-\lambda^{*}(\mathbf{x}) \hat{\psi}(\mathbf{x})|\alpha(\mathbf{x})\rangle\right],
\end{aligned}
$$

where $|\alpha(\mathbf{x})\rangle$ is a coherent state of polaritons at position $\mathbf{x}$ with complex amplitude $\alpha(\mathbf{x})$. Expectation values calculated with the Wigner distribution function correspond to expectation values of symmetrized operator expressions. For example for the one-body density matrix, we have

$$
\begin{aligned}
& \int d^{2} \psi(\mathbf{x}) P_{W}[\psi(\mathbf{x})] \psi^{*}(\mathbf{x}) \psi\left(\mathbf{x}^{\prime}\right) \\
& =\frac{1}{2} \operatorname{Tr}\left\{\rho\left[\hat{\psi}^{\dagger}(\mathbf{x}) \hat{\psi}\left(\mathbf{x}^{\prime}\right)+\hat{\psi}\left(\mathbf{x}^{\prime}\right) \hat{\psi}^{\dagger}(\mathbf{x})\right\} .\right.
\end{aligned}
$$

The operation of the creation and annihilation fields on the density matrix can be translated into the following functional operations on the Wigner distribution: ${ }^{37}$

$$
\begin{gathered}
\hat{\psi}(\mathbf{x}) \rho \rightarrow\left(\psi(\mathbf{x})+\frac{1}{2} \frac{\partial}{\psi(\mathbf{x})}\right) P_{W}\left[\psi(\mathbf{x}), \psi^{*}(\mathbf{x})\right], \\
\rho \hat{\psi}(\mathbf{x}) \rightarrow\left(\psi(\mathbf{x})-\frac{1}{2} \frac{\partial}{\psi(\mathbf{x})}\right) P_{W}\left[\psi(\mathbf{x}), \psi^{*}(\mathbf{x})\right], \\
\hat{\psi}^{\dagger}(\mathbf{x}) \rho \rightarrow\left(\psi(\mathbf{x})-\frac{1}{2} \frac{\partial}{\psi(\mathbf{x})}\right) P_{W}\left[\psi(\mathbf{x}), \psi^{*}(\mathbf{x})\right], \\
\rho \hat{\psi}^{\dagger}(\mathbf{x}) \rightarrow\left(\psi(\mathbf{x})+\frac{1}{2} \frac{\partial}{\psi(\mathbf{x})}\right) P_{W}\left[\psi(\mathbf{x}), \psi^{*}(\mathbf{x}) .\right]
\end{gathered}
$$

With these operator correspondences, the equation of motion for the Wigner quasiprobability distribution $P_{W}$ is obtained from the master equation (21):

$$
\begin{aligned}
\frac{\partial P_{W}\left[\psi(\mathbf{x}), \psi^{*}(\mathbf{x})\right]}{\partial t} & \left\{\frac{\partial}{\partial \psi(\mathbf{x})} F_{\mathrm{det}}-\frac{\partial}{\partial \psi^{*}(\mathbf{x})} F_{\text {det }}^{*}\right. \\
& +\frac{\partial^{2}}{\partial \psi(\mathbf{x}) \psi^{*}(\mathbf{x})}\left[\gamma+\mathcal{R}_{\text {in }}+\mathcal{R}_{\text {out }}\right]+i \frac{g}{2 \Delta V} \frac{\partial^{2}}{\partial \psi(\mathbf{x}) \psi^{*}(\mathbf{x})} \\
& \left.\times\left[\frac{\partial}{\partial \psi^{*}(\mathbf{x})} \psi^{*}(\mathbf{x})-\frac{\partial}{\partial \psi(\mathbf{x})} \psi(\mathbf{x})\right]\right\} P_{W}\left[\psi(\mathbf{x}), \psi^{*}(\mathbf{x})\right],
\end{aligned}
$$

where $F_{\text {det }}$ is the deterministic mean-field force acting on the polaritons,

$$
F_{\mathrm{det}}=-i\left[\epsilon_{\mathrm{LP}}(-i \nabla)+\frac{i\left(\mathcal{R}_{\mathrm{in}}-\mathcal{R}_{\mathrm{out}}-\gamma\right)}{2}+\frac{g}{\Delta V}|\psi(\mathbf{x})|^{2}\right] \psi(\mathbf{x}) .
$$

In Eq. (28), a momentum cutoff for the field $\psi$ is implicitly introduced by formulating the problem on a spatial grid with cell area $\Delta V$. The expression $\mathcal{R}_{\text {in,out }} \psi$ should be understood as

$$
\mathcal{R}_{\text {in }} \psi(\mathbf{x})=n_{R}^{2}(\mathbf{x}) \sum_{\mathbf{q}, \mathbf{x}^{\prime}} e^{i \mathbf{q}\left(\mathbf{x}-\mathbf{x}^{\prime}\right)} R_{\text {in }}\left[\epsilon_{\mathrm{LP}}(\mathbf{q})\right] \psi\left(\mathbf{x}^{\prime}\right),
$$




$$
\mathcal{R}_{\text {out }} \psi(\mathbf{x})=n_{R}(\mathbf{x}) \sum_{\mathbf{q}} e^{i \mathbf{q}\left(\mathbf{x}-\mathbf{x}^{\prime}\right)} R_{\text {out }}\left[\epsilon_{\mathrm{LP}}(\mathbf{q})\right] \psi\left(\mathbf{x}^{\prime}\right) .
$$

From the mathematical point of view, the last term in the equation of motion [Eq. (28)] has proved to make it very hard to find numerical approximations to the solutions of Eq. (28). If this term is neglected (the so-called truncated Wigner approximation), the quasiprobability distribution $P_{W}$ obeys a standard Fokker-Planck equation that corresponds to the Langevin equation,

$$
d \psi(\mathbf{x})=F_{\operatorname{det}}\left[\psi(\mathbf{x}), \psi^{*}(\mathbf{x})\right]+d W(\mathbf{x}),
$$

where $d W$ is a complex Gaussian stochastic variable with the correlation functions:

$$
\begin{gathered}
\left\langle d W(\mathbf{x}) d W\left(\mathbf{x}^{\prime}\right)\right\rangle=0, \\
\left\langle d W(\mathbf{x}) d W^{*}\left(\mathbf{x}^{\prime}\right)\right\rangle=\frac{d t}{2 \Delta V}\left(\left\langle\mathbf{x}\left|\mathcal{R}_{\text {in }}^{S}+\mathcal{R}_{\text {out }}^{S}\right| \mathbf{x}^{\prime}\right\rangle+\gamma \delta_{\mathbf{x}, \mathbf{x}^{\prime}}\right),
\end{gathered}
$$

where $\mathcal{R}_{\text {in,out }}^{S}=\left[\mathcal{R}_{\text {in,out }}+\left(\mathcal{R}_{\text {in,out }}\right)^{\mathrm{T}}\right] / 2$ are the symmetrized kernels. Solutions to the Langevin equation can be obtained by standard stochastic numerical techniques.

Let us now estimate the order of magnitude of the thirdorder derivative in Eq. (28) with respect to the other terms, in particular the second-order derivative terms. The function $P_{W}$ is peaked around the value of the field $\psi(x)$ whose squared modulus equals $|\psi(\mathbf{x})|^{2}=N(\mathbf{x})+1 / 2$. The variation in $P_{W}$ occurs on a scale of its argument of order one. Derivatives are therefore expected to be of the order of the function $P_{W}$ itself and the prefactors determine the relative importance of the derivative terms in Eq. (4). This leads us to the conclusion that the third-order derivative is negligible with respect to the second-order one if

$$
\gamma \gg \frac{g}{\Delta V} .
$$

The dissipative character of the system thus increases the region of validity of the truncated Wigner approximation. The dissipation gives away information about the system and destroys nontrivial quantum states (e.g., number or Schrödinger cat states). In terms of the Wigner function, oscillations of $P_{W}$ accompanied by regions where it becomes negative (that cannot be represented by a regular probability distribution) are washed out by the dissipation. ${ }^{38}$ Nontrivial quantum states are actually not expected to be relevant in polariton condensation but rather thermal and coherent states. These have a well-behaved Wigner quasiprobability distribution that can in principle be sampled by a Langevin equation. Considering the case of a stable gas $\left(R_{\text {in }}=R_{\text {out }}=\gamma\right.$ $=0$ ) reveals however that a thermal/coherent state is not always well described by the truncated Wigner approximation. When there is no dissipation, the second-order derivative in Eq. (28) is absent and neglecting the third-order derivative in Eq. (28) can only be justified with respect to the first-order one. The approximation then only holds in the regime when each grid cell is occupied by a large number of particles. ${ }^{26}$ Under this condition, spontaneous scattering (a quantum ef- fect not easy to describe correctly within classical field theory) is dominated by stimulated scattering. In momentum space, such a model is truncated at a momentum where the difference between the classical (Rayleigh-Jeans) and quantum (Bose-Einstein) momentum distributions is small. In the presence of dissipation on the other hand, spontaneous scattering from the reservoir into the classical field is described by the second-order derivatives and turns out to be taken correctly into account by the Langevin Eq. (32), and it becomes possible to obtain a reliable description of all the modes that have an appreciable occupation. We will revisit this issue when we relate our formalism to the Boltzmann equation in Sec. VI.

\section{EXCITON RESERVOIR}

In our description of the microcavity dynamics, the excitonlike particles are treated as a classical reservoir. This approximation allowed to trace out the excitonic degrees of freedom and to isolate the quantum dynamics of the polaritons from the classical exciton dynamics. In principle, the reservoir density appears as a deterministic classical quantity in the resulting equations of motion for the lower polariton dynamics. Physically, however, this is not expected to be a very good approximation because the condensate serves as a relaxation mechanism for the excitons. Stimulated scattering makes the rate of this relaxation to depend on the condensate population. We propose to go beyond the approximation that the reservoir is unaltered by the system by coupling its dynamics to the equation of motion for the classical polariton field

$$
\frac{d n_{R}}{d t}=-\gamma_{R}\left[n_{R}-n_{R}^{o}\left(I_{p}, \psi\right)\right] .
$$

Similar ideas have been implemented in Ref. 22, where the dynamics of a single condensate mode was coupled to a Boltzmann equation for the excited states, and in Ref. 5, where the reservoir was modeled by a saturable gain medium, a model widely used in laser physics. ${ }^{39}$

In Eq. (35), $n_{R}^{o}\left(I_{p}, \psi\right)$ is the average steady-state value of the reservoir density in the presence of a pump with intensity $I_{p}$ and a lower polariton field $\psi$. The relaxation time $\gamma_{R}^{-1}$ is a measure of the time it takes for the reservoir density to adjust to a new environment $\left(I_{p}, \psi\right)$. Spatial diffusion of the reservoir excitons is expected to be a small effect ${ }^{25}$ and was therefore neglected. For the steady-state value of the reservoir density, we assume that it is simply proportional to the balance of incoming and outgoing particles,

$$
n_{R}^{o}(P, \psi)=\beta\left(I_{p}-\left.\frac{d}{d t}\left\langle\psi^{\dagger} \psi\right\rangle\right|_{\mathrm{res}}\right),
$$

where $\left.\frac{d}{d t}\left\langle\psi^{\dagger} \psi\right\rangle\right|_{\text {res }}=2 \operatorname{Re}\left[\psi^{*}\left(\mathcal{R}_{\text {in }}-\mathcal{R}_{\text {out }}\right) \psi\right]$ is the net scattering rate from the reservoir into the lower polariton branch. It is instructive to substitute Eq. (36) into Eq. (35):

$$
\frac{d n_{R}}{d t}=P-\gamma_{R} n_{R}-\left.\beta \gamma_{R} \frac{d}{d t}\left\langle\psi^{\dagger} \psi\right\rangle\right|_{\mathrm{res}},
$$

where $P=\beta I_{p}$ is the effective pump term for the active reservoir polaritons. The parameter $\beta$ quantifies the backaction 
of the condensate on the reservoir. This backaction is needed to obtain a steady state for the dynamical equations above the threshold, where for $n_{R}=P / \gamma_{R}$ the inscattering rate exceeds the outscattering rate. In mean-field theory, the reservoir density $n_{R}$ is clamped to its threshold value $n_{R, \mathrm{mf}}$ that satisfies for homogeneous systems $n_{R, \mathrm{mf}}^{2} R_{\text {in }}(0)-n_{R, \mathrm{mf}} R_{\text {out }}(0)$ $=\gamma$. If we rewrite the motion equations for $n_{R}$ in terms of the renormalized $\tilde{n}_{R}=n_{R} / n_{R, \mathrm{mf}}$, we have

$$
\frac{d \tilde{n}_{R}}{d t}=\tilde{P}-\gamma_{R} \tilde{n}_{R}-\left.\alpha \frac{d}{d t}\left\langle\psi \psi^{\dagger} \psi\right\rangle\right|_{\text {res }},
$$

where $\alpha=\beta \gamma_{R} / n_{R \text {, mf }}$. Also in the presence of fluctuations, the dimensionless reservoir density $\tilde{n}_{R}$ is close to one above threshold, in order for the gain to compensate for the losses. The factor $\alpha$ plays an important physical role because the backaction of the condensate on the reservoir tends to damp the condensate fluctuations. If the condensate density is at some time larger than average, the reservoir will be depleted; $R_{\text {in }}-R_{\text {out }}$ decreases and the deterministic part in the equations of motion for the condensate will decrease the amplitude of the fluctuation. In principle, the parameter $\alpha$ could be calculated from the Boltzmann equation. We prefer however to study the physics in terms of this parameter because it gives a good insight in the nonequilibrium aspects of the coherence.

In the truncated Wigner approximation, the density of polaritons is related to $\psi$ as $n=|\psi|^{2}-1 /(2 \Delta V)$, or in words, the classical field $\psi$ contains half a particle per mode of zeropoint fluctuations. These fluctuations should be taken into account when evaluating the last term in Eq. (38). For the outscattering, the zero-point fluctuations do not contribute and should be subtracted, whereas for the inscattering, the zero-point fluctuations give rise to only half of the spontaneous inscattering. The remaining part should be added. The equation of motion for the reduced reservoir density then finally reads as

$$
\begin{aligned}
\frac{d \tilde{n}_{R}}{d t}= & \widetilde{P}-\gamma_{R} \tilde{n}_{R}-\left.\alpha \frac{d}{d t}\left\langle\psi^{\dagger} \psi\right\rangle\right|_{\text {res }, W} \\
& -\frac{\alpha}{2 \Delta V} \sum_{\mathbf{k}}\left\{R_{\text {in }}\left[\epsilon_{\mathrm{LP}}(\mathbf{k})\right]+R_{\text {out }}\left[\epsilon_{\mathrm{LP}}(\mathbf{k})\right]\right\} .
\end{aligned}
$$

\section{RELATION WITH THE BOLTZMANN EQUATION}

In the dissipative case, the derivation of the truncated Wigner equation did not rely on the formation of a condensate. We can therefore describe within the same formalism the condensed and noncondensed polariton gases. In the case that coherence is negligible, it is instructive to simplify the stochastic equations of motion Eq. (32). We will find that in the incoherent regime, the polariton condensate can be described with a Boltzmann-type equation. ${ }^{40}$

For simplicity, we consider the case of a uniform reservoir density. By writing the stochastic motion equations for the field $\psi(\mathbf{k})$ in momentum space, treating the interactions in the second Born approximation and assuming that there are no phase relations between the different momentum compo- nents $\left\langle\psi^{*}(\mathbf{k}, t) \psi\left(\mathbf{k}^{\prime}, t\right)\right\rangle=[N(\mathbf{k}, t)+1 / 2] \delta_{\mathbf{k}, \mathbf{k}^{\prime}}$, one obtains the following Boltzmann-type equation for the time evolution of the densities in momentum space,

$$
\begin{aligned}
\frac{d N(\mathbf{k}, t)}{d t}= & R_{\mathrm{in}}\left[\epsilon_{\mathrm{LP}}(\mathbf{k})\right][N(\mathbf{k}, t)+1]-\left\{R_{\mathrm{out}}\left[\epsilon_{\mathrm{LP}}(\mathbf{k})\right]+\gamma\right\} N(\mathbf{k}, t) \\
& +I_{B}[N(\mathbf{k}, t)]+I_{C}[N(\mathbf{k}, t)] .
\end{aligned}
$$

The first two terms describe the evolution of the mode occupation due to the interaction with the reservoir and losses through the cavity mirrors. Collisions are described by the last two terms. $I_{B}$ is the usual Boltzmann collision integral,

$$
\begin{aligned}
I_{B}\left[N_{1}\right]= & -2 \pi g^{2} \sum_{\mathbf{k}_{1}, \mathbf{k}_{2}} \delta_{\gamma-R}\left(\epsilon_{1}+\epsilon_{2}-\epsilon_{3}-\epsilon_{4}\right) \\
& \times\left[N_{1} N_{2}\left(1+N_{3}\right)\left(1+N_{4}\right)-N_{3} N_{4}\left(1+N_{1}\right)\left(1+N_{2}\right)\right],
\end{aligned}
$$

where $N_{1}(t)=N(\mathbf{k}, t), N_{2}(t)=N\left(\mathbf{k}_{1}+\mathbf{k}_{2}-\mathbf{k}, t\right), N_{3}(t)=N\left(\mathbf{k}_{1}, t\right)$, $N_{4}(t)=N\left(\mathbf{k}_{2}, t\right)$ and analogous for the energies $\epsilon_{i}$. The $\delta$ function for energy conservation is broadened due to the finite lifetime of the polaritons to a Lorentzian with width $\left(\gamma-R_{\text {in }}\right.$ $\left.+R_{\text {out }}\right)$.

The extra collisional term is due to the fact that our stochastic classical field model does not coincide with the true quantum dynamics (the third-order derivatives in Eq. (28) are neglected):

$$
\begin{aligned}
I_{C}\left[N_{1}\right]= & -\frac{\pi g^{2}}{2} \sum_{\mathbf{k}_{1}, \mathbf{k}_{2}} \delta_{\gamma-R}\left(\epsilon_{1}+\epsilon_{2}-\epsilon_{3}-\epsilon_{4}\right) \\
& \times\left[N_{1}+N_{2}-N_{3}-N_{4}\right] .
\end{aligned}
$$

This term is spurious because the Boltzmann equation should be recovered in the incoherent limit. Our classical field model can therefore only be a good approximation of the full quantum dynamics if the term $I_{C}$ is negligible with respect to the other terms in Eq. (40). It scales as $I_{C} \propto(g / \Delta V)(g n \Delta V)$. If the occupation numbers per grid cell $n \Delta V$ are much larger than unity, the Boltzmann collision term $I_{B}$ is obviously dominant with respect to $I_{C}$. This is the typical condition for the use of the Wigner distribution function in the description of a stable Bose gas. ${ }^{26}$ For bosons with a finite lifetime, this condition can be relaxed because even when $n \Delta V$ is not much larger than unity, the spurious term can be still much smaller than the reservoir term $R_{\text {in }}$. The inscattering rate $R_{\text {in }}$ should compensate the losses $\gamma$. If occupation numbers are not large, the truncated Wigner is therefore still expected to yield physical results if $g / \Delta V \ll \gamma$. Note that the latter requirement coincides with condition (34) derived from the full equation of motion Eq. (28).

If we neglect the collisional terms in Eq. (40), the steadystate solution is

$$
N(\mathbf{k})=\frac{1}{\left[\gamma+R_{\text {out }}\left(\epsilon_{\mathrm{k}}\right)\right] / R_{\text {in }}\left(\epsilon_{\mathrm{k}}\right)-1} .
$$

The simplest model that yields a temperature $T_{R}$ (that is in experiments typically higher than the lattice temperature) for the tail of the polariton momentum distribution is obtained by setting the outscattering rate to zero, 


$$
\begin{gathered}
R_{\text {out }}\left(\widetilde{n}_{R}, E\right)=0, \\
R_{\text {in }}\left(\widetilde{n}_{R}, E\right)=\gamma \widetilde{n}_{R}^{2} \exp \left(-E / T_{R}\right) .
\end{gathered}
$$

The inscattering rate is fixed by the definition that threshold is reached at $\tilde{n}_{R}=1$. Studies of the Boltzmann equation ${ }^{41}$ have however shown that the rates $R_{\text {in }}$ and $R_{\text {out }}$ both tend to increase as a function of the energy, approximately as

$$
\begin{gathered}
R_{\text {in }}\left(\tilde{n}_{R}, E\right)=(1+\eta) \gamma \exp \left(E / k_{B} T_{R}\right), \\
R_{\text {out }}\left(\tilde{n}_{R}, E\right)=\eta \gamma \exp \left(2 E / k_{B} T_{R}\right),
\end{gathered}
$$

where $\eta$ is a dimensionless parameter that quantifies the outscattering. We will see below that a nonzero outscattering enhances fluctuations.

\section{NUMERICAL RESULTS}

The stochastic motion equations can be simulated by Monte Carlo techniques. As we have already mentioned in Sec. I, the nonequilibrium condition of the polariton condensates makes that the effect of the reservoir on the correlation functions cannot be characterized by the temperature alone. We will discuss below two other physical quantities that determine the degree of coherence in the polariton condensate: the feedback parameter $\alpha$ and the outscattering rate $R_{\text {out }}$. The other parameters we keep fixed for all simulations are the following: $\quad \gamma=0.5 \mathrm{meV}, \quad m_{\mathrm{LP}} / \hbar=1 \quad \mu \mathrm{m}^{-2} \mathrm{meV}^{-1}, \quad g / \hbar$ $=0.03 \mu \mathrm{m}^{2}$, and $k_{B} T_{R}=2 \mathrm{meV}$. The simulations were done on a $32 \times 32$ point grid with physical dimension of $66 \times 66 \mu \mathrm{m}^{2}$ and periodic boundary conditions. The deterministic evolution of the polariton field [Eq. (29)] was calculated with a split-step method, evaluating the kinetic energy in momentum space and the other terms in real space and transforming back and forth using fast Fourier transform (FFT) algorithms. The stochastic fields with the spatial correlation functions given by Eq. (33) were constructed from unit variance uncorrelated complex Gaussian noise fields $\xi(\mathbf{x})$ by the linear transformation $d \mathbf{W}=\mathbf{D} \xi$, where we used a vector notation for the noise fields and $\mathbf{D}$ is the matrix square root of the noise correlation $\mathbf{D}=\sqrt{\left\langle d \mathbf{W} d \mathbf{W}^{\dagger}\right\rangle}$. The calculation of this square root is an important addition to the numerical complexity in computing the time evolution of the lower polariton field.

Figures 2 and 3 illustrate single Monte Carlo realizations of the classical field $\psi(\mathbf{x})$. Even though these images have strictly speaking no direct physical meaning, they already illustrate qualitatively the coherence properties of the polariton condensate. Figure 2 shows two examples for a finite excitation spot for pump intensities below (panels a and b) and above the threshold (panels c and d). At low density, both the density and phase fluctuations are large, whereas the phase fluctuations are clearly suppressed in the high-density regime. Panel (d) shows that phase coherence exists all over the extent of the excitation region. The concentric phase profile originates from the repulsive polariton-polariton interaction that causes an outward flow of polaritons. ${ }^{10}$

Figure 3 shows snapshots of the polariton density and phase for a uniform pump below and slightly above the
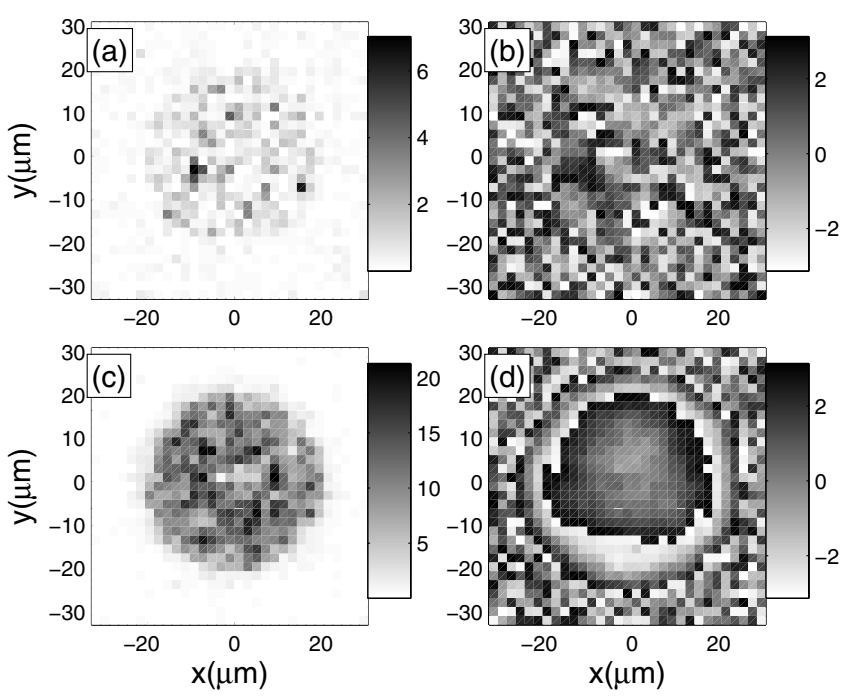

FIG. 2. Snapshots of a single Monte Carlo realization of [(a) and (c)] the density and [(b) and (d)] phase for excitation parameters [(a) and (b)] below and [(c) and (d)] above threshold in the case of a finite excitation spot.

threshold. The phase profile of panel (d) shows that the phase ordering is only partial. Vortex-antivortex pairs appear to exist at densities well above the stimulation threshold. This is an indication that the physics of the Berezinskii-KosterlitzThouless type $\mathrm{e}^{7,13}$ could occur in polariton condensates.

Three momentum distributions for increasing pump intensity are shown in Fig. 4. As expected, our model shows the buildup of a large occupation of the low-momentum states for increasing pump intensity. The momentum distributions appear to be rather well fitted by a Bose-Einstein function (full line). It is important to mention here the important role of the reservoir relaxation rates. We have chosen them in such a way that a thermal distribution is obtained even in the
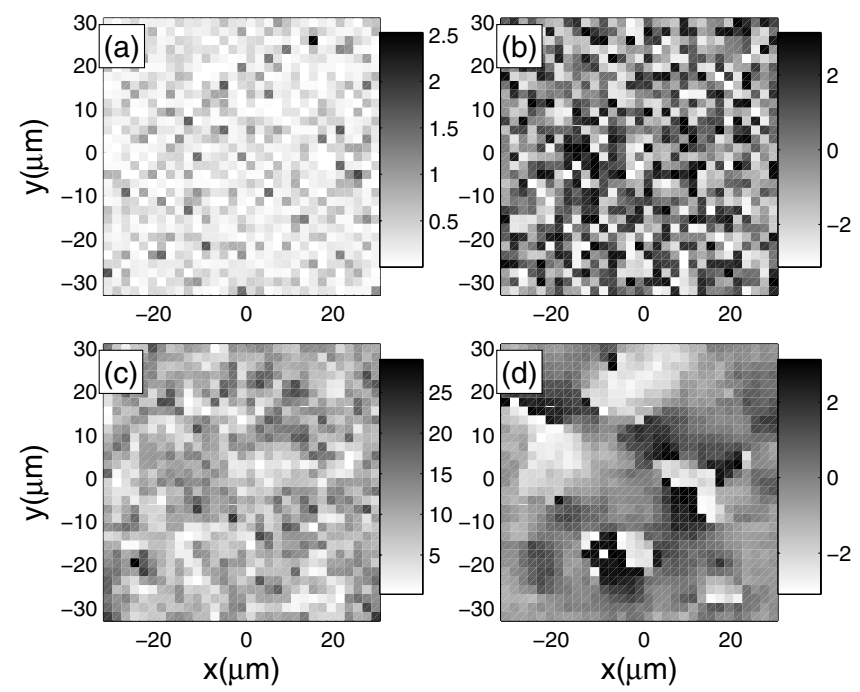

FIG. 3. Snapshots of a single Monte Carlo realization of [(a) and (c)] the density and [(b) and (d)] phase for excitation parameters [(a) and (b)] below and [(c) and (d)] above threshold of a polariton condensate excited by a uniform excitation laser with periodic boundary conditions. 

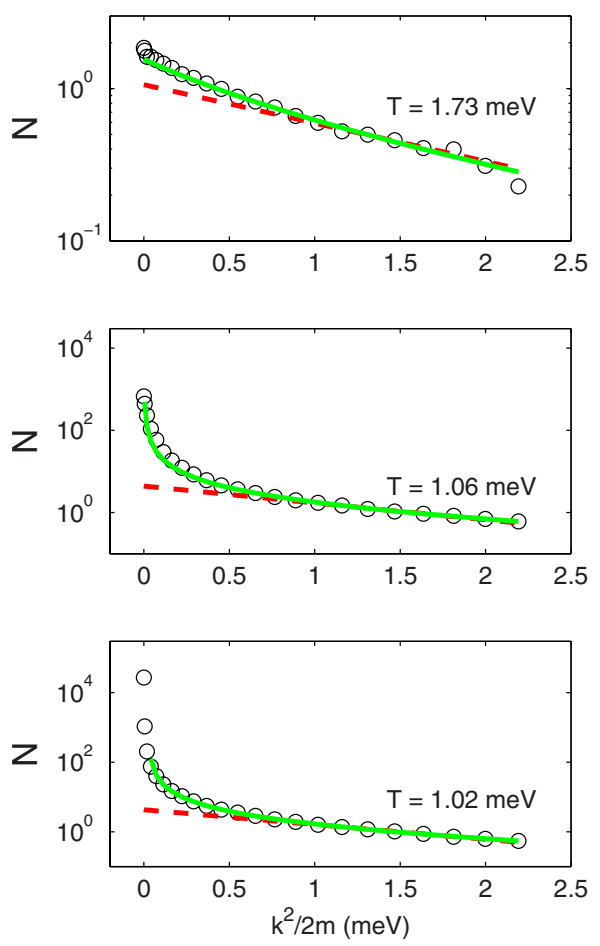

FIG. 4. (Color online) Momentum distribution of the polariton field for various pump intensities. The open circles are the result of the Monte Carlo simulations and the full line is a fit to a BoseEinstein distribution. Statistical errors of the Monte Carlo simulations are within the symbol size.

absence of collisions between lower polaritons. In simulations with energy-independent relaxation rates (not shown) and a large yet realistic ${ }^{42}$ polariton-polariton interaction strength, we have obtained a constant instead of exponential decay at large momenta.

Note that the temperature extracted from the fits of the tails to a Maxwellian is lower than the reservoir temperature $T_{R}$ (2 meV for the present simulations) that enters the rates $R_{\text {in,out }}$ according to Eq. (47): the nonlinearity modifies the temperature that is expected in the linear regime. We remind the reader that $T_{R}$ does not coincide with the lattice temperature and that $T_{\mathrm{fit}}<T_{R}$ does not imply that the polariton temperature is lower than the lattice temperature.

The subtle features of long-range coherence are much clearer in the Fourier transform of the momentum distribution, i.e., the first-order spatial coherence function. In Fig. 5 two values of $\alpha$ are compared. Below the condensation threshold, the gain saturation parameter $\alpha$ [see Eq. (39)] has no influence and the fit of the coherence by the $g^{(1)}$ of the noninteracting Bose gas yields the reservoir temperature of 2 meV. For the simulation above the threshold, a higher value of $\alpha$ improves the long-range coherence. Both spatial coherence functions are relatively well fitted by one of a noninteracting Bose gas. Both temperatures are below the reservoir temperature. The lowest effective temperature is obtained for the largest feedback parameter $\alpha$.

In the simulations of Fig. 5, the outscattering was set to zero. In the simulations presented in Fig. 6, we have included this effect. In order to avoid exceedingly large rates in the model, we have put a cutoff in the magnitude of $R_{\text {out }}$ as
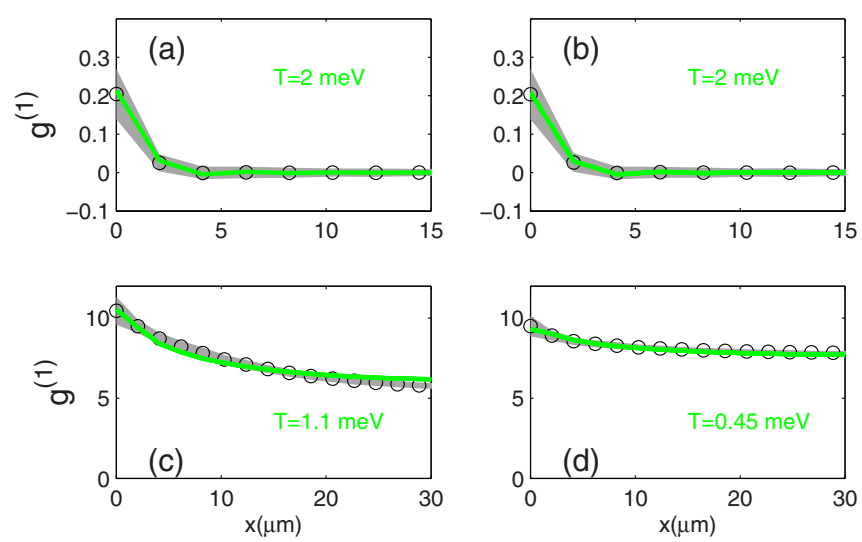

FIG. 5. (Color online) (c, d) Spatial coherence (open circles, the gray band indicates the error on the Monte Carlo data) for two values of the feedback from the condensate on the reservoir: from left to right [(a) and (c)] $\alpha=0.01 \mu \mathrm{m}^{2}$ and [(b) and (d)] $\alpha$ $=0.1 \mu \mathrm{m}^{2}$. The full line shows the decay of the correlations in a noninteracting Bose gas at the temperature reported in the panels.

$R_{\text {out }}(E)=\min \left[2 \gamma e^{2 E / T_{R}}, 3.3 \mathrm{meV}\right]$. The inscattering rate was chosen $R_{\text {in }}(E)=\left[R_{\text {out }}(E)+\gamma\right] e^{-E / T_{R}}$.

Figure 6 shows that the outscattering has a big effect on the coherence function. This should not come as a surprise because the outscattering increases the fluctuations (physically shot noise due to the discrete nature of the polariton field). Keeping $\alpha=0.01 \mu \mathrm{m}^{-1}$ as in Fig. 5 but including some outscattering, the coherence in panel (a) is dramatically decreased. As compared to the simulations of Fig. 5, the effect of $\alpha$ is much more pronounced. For the smallest value of $\alpha$, the temperature of $5.5 \mathrm{meV}$ for which the spatial coherence is reasonably well fitted is much larger than the one that is extracted from the tail of the momentum distribution (less than $1 \mathrm{meV}$ ): the polariton condensate behaves in this regime very different from the ideal Bose gas.

Another quantity of great physical interest is the secondorder coherence function $g^{(2)}\left(\mathbf{x}, t ; \mathbf{x}^{\prime}, t^{\prime}\right)$ $=\left\langle\psi^{\dagger}(\mathbf{x}, t) \psi^{\dagger}\left(\mathbf{x}^{\prime}, t^{\prime}\right) \psi\left(\mathbf{x}^{\prime}, t^{\prime}\right) \psi(\mathbf{x}, t)\right\rangle$ that quantifies the density fluctuations. Experimentally, the equal position second-order coherence was investigated in Ref. 17 for equal times $t=t^{\prime}$ and in Ref. 5 as a function of the delay $t-t^{\prime}$. Within the Wigner formalism, different time correlation functions are not straightforwardly calculable, so we present here only results for the equal time second-order coherence.

Results of the equal position second-order coherence $g^{(2)}(0) \equiv g^{(2)}(\mathbf{x}, t ; \mathbf{x}, t)$ are shown in Fig. 7 for several parameter values. As expected, $g^{(2)}(0)$ approaches the value 2 of
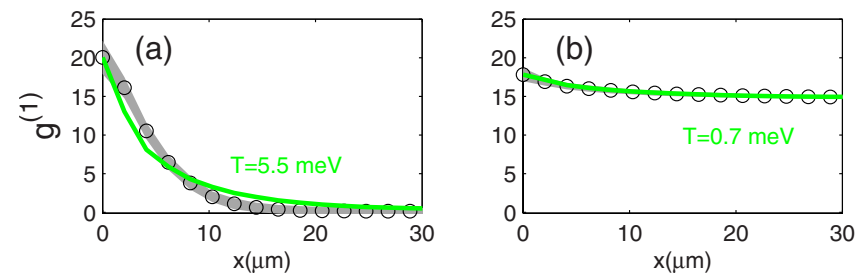

FIG. 6. (Color online) The spatial coherence function as in Fig. 5, but including outscattering. Simulations with (a) $\alpha=0.01 \mu \mathrm{m}^{2}$ and (b) $\alpha=0.1 \mu \mathrm{m}^{2}$ are shown. 


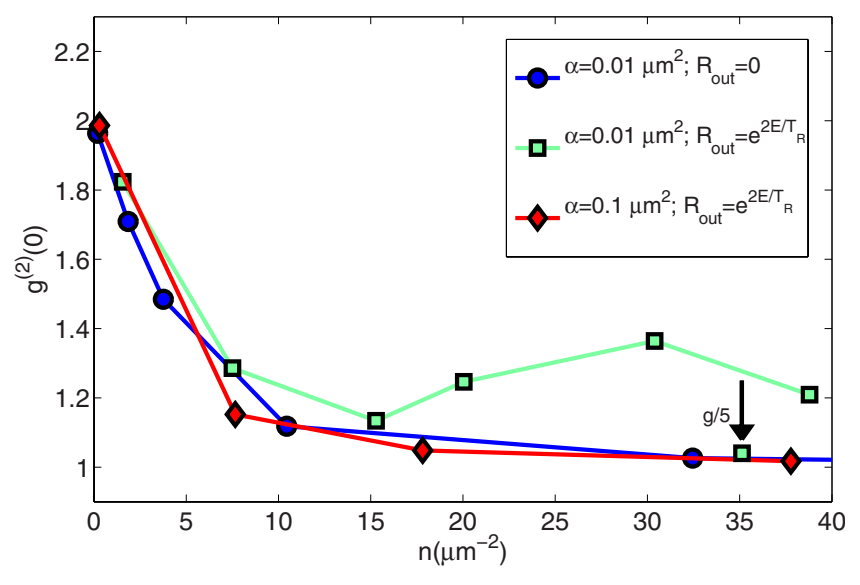

FIG. 7. (Color online) Second-order coherence function as a function of the total density obtained for different excitation powers and the simulation parameters indicated in the legend.

the incoherent Bose gas in the low-density regime. For increasing polariton densities, the second-order coherence decreases, but its actual value depends again strongly on the chosen parameter values. A larger value of the feedback parameter $\alpha$, suppresses the density fluctuations. This is in agreement with the model described in Ref. 5, where the density fluctuations are proportional to the saturation density (large saturation density means small feedback from the condensate on the reservoir). Figure 7 also shows that the outscattering increases the density fluctuations. This dependence is expected because adding the knock out processes leaves the deterministic term in the evolution equation for the classical field unaltered but increases the fluctuations.

Note that the density fluctuations are within our model, not always monotonous, but for some parameter values show a minimum value slightly above threshold. Nonmonotonous behavior of $g^{(2)}(0)$ was also observed in experiments on polariton condensation in CdTe microcavities. ${ }^{17}$ Also in the theoretical work of Refs. 43 and 44 based on a Boltzmann equation for the excited states coupled to a master equation for the condensate mode, an increase in density fluctuations above the threshold was found. It is however important to mention that in Fig. 7 the interaction energy is very large when $g^{(2)}(0)$ increases again $(1 \mathrm{meV}$ blueshift due to condensate-condensate interactions alone). When the value of the blueshift is reduced to $0.2 \mathrm{meV}, g^{(2)}(0)$ is found to be very close to one.

We want to point out that we have not found any regime with good long-range spatial coherence and large density fluctuations. Indeed, Fig. 6(a) shows that at the density $n$ $\approx 20 \mu \mathrm{m}^{-2}$ the spatial coherence is, although longer than the thermal de Broglie wavelength corresponding to $T_{R}$, limited to about $10 \mu \mathrm{m}$. Physically it is actually not expected that good spatial coherence and strong density fluctuations can go together because phase fluctuations are coupled to the density fluctuations through the interaction and kinetic energy. So far, in the experiments on CdTe microcavities where the increase in $g^{(2)}(0)$ as a function of pump power was observed, no decrease in spatial coherence was seen. It is possible that the distance at which the spatial coherence was probed is too short for the decrease in spatial coherence to be detectable, but we cannot exclude other explanations in terms of extrinsic experimental effects. The measured density fluctuations could, for example, contain a component due to intensity fluctuations in the excitation laser.

\section{CONCLUSIONS}

We have derived classical field equations for a nonresonantly excited polariton condensate in a semiconductor microcavity within the truncated Wigner approximation. Thanks to the polariton losses our model remains physical in the low-density regime and allows us to describe the polariton condensate at all densities. Our equations were shown to reduce to the Boltzmann equation in the low-density regime below threshold. Above threshold, the equations were analyzed numerically with Monte Carlo simulations. The firstand second-order spatial coherences were shown to depend dramatically on the feedback from the condensate on the reservoir (the gain saturation) and on the collisions with reservoir excitons that knock polaritons out of the condensate. Within our model, the density fluctuations can show nonmonotonous behavior as a function of the polariton density. We predict that an increase in density fluctuations is accompanied by a decrease in the spatial coherence.

Finally, the vortex defects in individual Monte Carlo realizations of the polariton field show that the spatial coherence is limited by the spontaneous appearance of vortex defects in the phase. A further study of the role of vortices is necessary to understand their effect on the spatial coherence.

\section{ACKNOWLEDGMENTS}

It is a pleasure to acknowledge numerous stimulating and insightful discussions with I. Carusotto, D. Sarchi, K. Lagoudakis, M. Richard, and B. Pietka.

\footnotetext{
${ }^{1}$ For a review, see J. Keeling, F. M. Marchetti, M. H. Szymanska, and P. B. Littlewood, Semicond. Sci. Technol. 22, R1 (2007).

${ }^{2}$ H. Deng, D. Press, S. Gotzinger, G. S. Solomon, R. Hey, K. H. Ploog, and Y. Yamamoto, Phys. Rev. Lett. 97, 146402 (2006).

${ }^{3}$ J. Kasprzak, M. Richard, S. Kundermann, A. Baas, P. Jeambrun, J. M. J. Keeling, F. M. Marchetti, M. H. Szymanska, R. André, J. L. Staehli, V. Savona, P. B. Littlewood, B. Deveaud, and L. S.
}

Dang, Nature (London) 443, 409 (2006).

${ }^{4}$ R. Balili, V. Hartwell, D. Snoke, L. Pfeiffer, and K. West, Science 316, 1007 (2007).

${ }^{5}$ A. P. D. Love, D. N. Krizhanovskii, D. M. Whittaker, R. Bouchekioua, D. Sanvitto, S. A. Rizeiqi, R. Bradley, M. S. Skolnick, P. R. Eastham, and R. André, and L. S. Dang, Phys. Rev. Lett. 101, 067404 (2008). 
${ }^{6}$ D. J. Bishop and J. D. Reppy, Phys. Rev. Lett. 40, 1727 (1978).

${ }^{7}$ Z. Hadzibabic, P. Krüger, M. Cheneau, B. Battelier, and J. Dalibard, Nature (London) 441, 1118 (2006).

${ }^{8}$ M. Richard, J. Kasprzak, R. Romestain, R. André, and L. S. Dang, Phys. Rev. Lett. 94, 187401 (2005).

${ }^{9}$ M. Richard, J. Kasprzak, R. André, R. Romestain, L. S. Dang, G. Malpuech, and A. Kavokin, Phys. Rev. B 72, 201301(R) (2005).

${ }^{10}$ M. Wouters, I. Carusotto, and C. Ciuti, Phys. Rev. B 77, 115340 (2008).

${ }^{11}$ K. G. Lagoudakis, M. Wouters, M. Richard, A. Baas, I. Carusotto, R. André, L. S. Dang, and B. Deveaud-Plédran, Nat. Phys. 4, 706 (2008).

${ }^{12}$ J. Keeling and N. G. Berloff, Phys. Rev. Lett. 100, 250401 (2008).

${ }^{13}$ J. M. Kosterlitz and D. J. Thouless, J. Phys. C 6, 1181 (1973).

${ }^{14}$ D. S. Petrov, M. Holzmann, and G. V. Shlyapnikov, Phys. Rev. Lett. 84, 2551 (2000).

${ }^{15}$ H. Deng, G. S. Solomon, R. Hey, K. H. Ploog, and Y. Yamamoto, Phys. Rev. Lett. 99, 126403 (2007).

${ }^{16}$ H. Deng, G. Weihs, C. Santori, J. Bloch, and Y. Yamamoto, Science 298, 199 (2002).

${ }^{17}$ J. Kasprzak, M. Richard, A. Baas, B. Deveaud, R. André, J.-Ph. Poizat, and L. S. Dang, Phys. Rev. Lett. 100, 067402 (2008).

${ }^{18}$ F. Tassone and Y. Yamamoto, Phys. Rev. B 59, 10830 (1999).

${ }^{19}$ T. D. Doan, H. T. Cao, D. B. Tran Thoai, and H. Haug, Phys. Rev. B 72, 085301 (2005).

${ }^{20}$ G. Malpuech, A. Kavokin, A. Di Carlo, and J. J. Baumberg, Phys. Rev. B 65, 153310 (2002).

${ }^{21}$ I. Shelykh, G. Malpuech, K. V. Kavokin, A. V. Kavokin, and P. Bigenwald, Phys. Rev. B 70, 115301 (2004).

${ }^{22}$ F. P. Laussy, G. Malpuech, A. Kavokin, and P. Bigenwald, Phys. Rev. Lett. 93, 016402 (2004).

${ }^{23}$ D. Sarchi and V. Savona, Phys. Rev. B 75, 115326 (2007).

${ }^{24}$ M. H. Szymańska, J. Keeling, and P. B. Littlewood, Phys. Rev. Lett. 96, 230602 (2006).

${ }^{25}$ M. Wouters and I. Carusotto, Phys. Rev. Lett. 99, 140402 (2007).
${ }^{26}$ A. Sinatra, C. Lobo, and Y. Castin, J. Phys. B 35, 3599 (2002).

${ }^{27}$ H. T. C. Stoof and M. J. Bijlsma, J. Low Temp. Phys. 124, 431 (2001).

${ }^{28}$ C. W. Gardiner and P. Zoller, Phys. Rev. A 55, 2902 (1997).

${ }^{29}$ E. Zaremba, T. Nikuni, and A. Griffin, J. Low Temp. Phys. 116, 277 (1999).

${ }^{30}$ C. W. Gardiner and M. J. Davis, J. Phys. B 36, 4731 (2003).

${ }^{31}$ P. B. Blakie, A. S. Bradley, M. J. Davis, R. J. Ballagh, and C. W. Gardiner, Adv. Phys. 57, 363 (2008).

${ }^{32}$ I. Carusotto and C. Ciuti, Phys. Rev. B 72, 125335 (2005).

${ }^{33}$ L. Giorgetti, I. Carusotto, and Y. Castin, Phys. Rev. A 76, 013613 (2007).

${ }^{34}$ T. P. Simula, M. J. Davis, and P. B. Blakie, Phys. Rev. A 77, 023618 (2008).

${ }^{35}$ D. Bajoni, P. Senellart, A. Lemaître, and J. Bloch, Phys. Rev. B 76, 201305(R) (2007).

${ }^{36}$ For a discussion out of equilibrium, see, e.g., H. Haug and A.-P. Jauho, Quantum Kinetics in Transport and Optics of Semiconductors (Springer, New York, 1998), part II.

${ }^{37}$ C. W. Gardiner and P. Zoller, Quantum Noise (Springer-Verlag, Berlin, 2000).

${ }^{38}$ W. H. Zurek, Rev. Mod. Phys. 75, 715 (2003).

${ }^{39}$ See, e.g., H. Ghafouri-Shiraz The Principles of Semiconductor Laser Diodes and Amplifiers (Imperial College Press, London, 2004).

${ }^{40}$ Yu. M. Kagan, B. V. Svistunov, and G. V. Shlyapnikov, Sov. Phys. JETP 74, 279 (1992); Yu. M. Kagan and B. V. Svistunov, Phys. Rev. Lett. 79, 3331 (1997).

${ }^{41}$ D. Porras, C. Ciuti, J. J. Baumberg, and C. Tejedor, Phys. Rev. B 66, 085304 (2002).

${ }^{42}$ An experimental upper bound to the blueshift due to polaritonpolariton interaction is given by the total blueshift, which is less than $1 \mathrm{meV}$.

${ }^{43}$ P. Schwendimann and A. Quattropani, Phys. Rev. B 77, 085317 (2008).

${ }^{44}$ D. Sarchi, P. Schwendimann, and A. Quattropani, Phys. Rev. B 78, 073404 (2008). 